\title{
Autonomia intelectual do paciente: relação entre o contato e a busca por conhecimentos sobre determinada doença na Atenção Primária
}

\author{
Patient's intellectual autonomy: relationship between contact and the search for knowledge about a \\ particular disease in Primary Care
}

Autonomía intelectual del paciente: relación entre el contacto y la búsqueda de conocimiento sobre una determinada enfermedad en Atención Primaria

Recebido: 30/09/2021 | Revisado: 09/10/2021 | Aceito: 16/10/2021 | Publicado: 18/10/2021

\author{
Márcia Farsura de Oliveira \\ ORCID: https://orcid.org/0000-0001-8462-0431 \\ Faculdade Dinâmica do Vale do Piranga, Brasil \\ E-mail: mmfarsura@yahoo.com.br \\ Pollyanna Álvaro Ferreira Spósito \\ ORCID: https://orcid.org/0000-0003-0875-6227 \\ Faculdade Dinâmica do Vale do Piranga, Brasil \\ E-mail: pollysposito@gmail.com \\ Caroline Silva de Araujo Lima \\ ORCID: https://orcid.org/0000-0003-2537-292X \\ Faculdade Dinâmica do Vale do Piranga, Brasil \\ E-mail: carolinearaujo689@gmail.com \\ Marli do Carmo Cupertino \\ ORCID: https://orcid.org/0000-0001-5790-0622 \\ Faculdade Dinâmica do Vale do Piranga, Brasil \\ E-mail: marlicuppertino@gmail.com
}

\begin{abstract}
Resumo
A Organização Mundial de Saúde informa que a maioria da população mundial viverá 60 anos ou mais. Para que se tenha uma vida mais longa, com qualidade de vida, o fator saúde é imprescindível. Consequentemente, ter acesso a informações claras e confiáveis, sobre as doenças com as quais convive, é uma ferramenta importante para prevenção e terapêutica das doenças. Assim, objetivou-se pesquisar, através de aplicação de questionário, a temática de eleição, pela clientela, para a ação de rodas de conversa em um ambulatório público, e verificar se o contato com a doença, influencia no interesse de busca por conhecimentos sobre a mesma. A experiência ocorreu na sala de espera do Ambulatório Sette de Barros, após aprovação Ética - parecer 2.925.286. O questionário impresso foi aplicado para 378 pessoas, nos meses de setembro e novembro de 2018. A pesquisa identificou que: (i) com relação aos temas eleito para discussão o mais votado foi depressão com 292 votos; seguido por hipertensão arterial (261); dislipidemia (256) e insônia 247; (ii) a maioria dos entrevistados demonstrou ter contato com alguém que apresenta hipertensão arterial (311 respondentes), seguido de depressão (291 optantes), insônia (289) e diabetes (287). Infere-se que há uma relação positiva entre escolha pela temática e a vivência dos pacientes com as doenças, já que é mais recorrente que os indivíduos apresentem dúvidas sobre o tema e a Atenção Primária mostra-se como um dos locais de busca por informações seguras e acessíveis sobre temáticas relacionadas a saúde.
\end{abstract}

Palavras-chave: Atenção Primária à Saúde; Estratégia Saúde da Família; Educação em saúde.

\begin{abstract}
The World Health Organization reports that the majority of the world's population will live to be 60 years or more. In order to have a longer life, with quality of life, the health factor is essential. Consequently, having access to clear and reliable information about the diseases you live with is an important tool for the prevention and treatment of diseases. Thus, the objective was to research, through the application of a questionnaire, the theme of choice, by the clientele, for the action of conversation circles in a public clinic, and to verify whether contact with the disease influences the interest in the search for knowledge about the same. The experience took place in the waiting room of the Sette de Barros Ambulatory, after Ethics approval - opinion 2,925,286. The printed questionnaire was applied to 378 people, in the months of September and November 2018. The survey identified that: (i) regarding the topics chosen for discussion, the most voted was depression with 292 votes; followed by arterial hypertension (261); dyslipidemia (256) and insomnia 247; (ii) most respondents demonstrated having contact with someone who has hypertension (311 respondents), followed by depression (291 opters), insomnia (289) and diabetes (287). It is inferred that there is a positive relationship between the choice for the topic and the experience of patients with the diseases, since it is more recurrent that
\end{abstract}


individuals have doubts about the topic and Primary Care is one of the places to search for safe information and accessible on health-related topics.

Keywords: Primary Health Care; Family Health Strategy; Health education.

\begin{abstract}
Resumen
La Organización Mundial de la Salud informa que la mayoría de la población mundial vivirá 60 años o más. Para tener una vida más larga, con calidad de vida, el factor salud es fundamental. En consecuencia, tener acceso a información clara y confiable sobre las enfermedades con las que vive es una herramienta importante para la prevención y el tratamiento de enfermedades. Assim, objetivou-se pesquisar, através de aplicação de questionário, a temática de eleição, pela clientela, para a ação de rodas de conversa em um ambulatório público, e verificar se o contato com a doença, influencia no interesse de busca por conhecimentos sobre La misma. La experiencia tuvo lugar en la sala de espera del Ambulatorio Sette de Barros, previa aprobación Ética - dictamen 2.925.286. El cuestionario impreso se aplicó a 378 personas, en los meses de septiembre y noviembre de 2018. La encuesta identificó que: (i) en cuanto a los temas elegidos para la discusión, el más votado fue la depresión con 292 votos; seguido de hipertensión arterial (261); dislipidemia (256) e insomnio 247; (ii) la mayoría de los encuestados demostró tener contacto con alguien que tiene hipertensión (311 encuestados), seguido de depresión (291 personas que eligieron), insomnio (289) y diabetes (287). Se infiere que existe una relación positiva entre la elección por el tema y la experiencia de los pacientes con las enfermedades, ya que es más recurrente que los individuos tengan dudas sobre el tema y la Atención Primaria es uno de los lugares para buscar información segura y accesible sobre temas relacionados con la salud.
\end{abstract}

Palabras clave: Atención Primaria de Salud; Estrategia de Salud de la Familia; Educación en salud.

\title{
1. Introdução
}

Mundialmente, na contemporaneidade, a maioria das pessoas possui expectativa de vida de 60 anos ou mais. Uma vida mais longa representa uma oportunidade, não apenas para o indivíduo e familiares, mas também para a sociedade como um todo. No entanto, essa oportunidade depende muito do fator saúde. Embora algumas das variações na saúde reflitam herança genética, a maior parte se deve ao ambiente físico e social. Tais ambientes começam a influenciar o envelhecimento desde a infância, por isso é necessário um cuidado constante em saúde, em busca de informações seguras e confiáveis sobre as doenças (OMS, 2017).

A confiança do público na profissão médica diminuiu nas últimas décadas. O livre acesso a informações sobre saúde disponíveis em várias fontes pode ser um fator contribuinte. Especificamente, a digitalização via mídia tradicional, por exemplo, jornal e televisão, diminuiu, mas aumentou nas novas mídias com mídias sociais e web. Além disso, tanto a busca quanto a discussão de informações sobre saúde caíram, no entanto, descobriu-se que a comunicação centrada no paciente é responsável por mediar as relações entre os tipos de aquisição de informações de saúde e a confiança do paciente nos médicos ao longo do tempo (Liu \& Jiang, 2019).

A alfabetização em saúde ${ }^{1}$ e o acesso a informações sobre saúde propiciam melhora na qualidade de vida do indivíduo e da população em geral (Oliveira \& Machado, 2020). As necessidades de informação de pessoas, que convivem com alguma patologia, seja ela de ordem física, social ou psicológica, não podem ser subestimadas com informações imprecisas e de qualidade duvidosa. Os profissionais de saúde precisam garantir que as necessidades de informação dos pacientes sejam atendidas na prática para que os pacientes se tornem ativamente envolvidos nas decisões de tratamento (Kugbey, Meyer-Weitz; Asante, 2019). Essa construção de conhecimentos conjunta, estabelecida entre profissionais de saúde e clientes, possibilita a maior autonomia dos sujeitos sobre as decisões quanto às terapêuticas, contribuindo, assim, para a integralidade da atenção, centrada no indivíduo, com sugestões democráticas, oportunizando o controle social e aumentando o compromisso com a cidadania (Ferreira et al., 2019A; Ferreira et al., 2019B; Liu \& Jiang, 2019).

A função da Estratégias Saúde da Família (ESF) é ser de caráter multiprofissional e interdisciplinar e responsável pela atenção integral à saúde da população, resgatando, por meio do atendimento humanizado, os vínculos de compromisso e

\footnotetext{
${ }^{1}$ Alfabetização em saúde é o produto - aquisição de conhecimentos e autonomia intelectual - das atividades de educação popular em saúde realizadas.
} 
corresponsabilidade entre os serviços de saúde, os profissionais e a população - que se dará, de forma mais eficaz, a partir da autonomia intelectual do paciente, tendo enfoque no fortalecimento da capacidade de respostas às doenças emergentes e endemias (Moretti-Pires \& Bueno, 2009). As informações fornecidas pela ESF, não podem ser baseadas nos saberes populares, pois, dessa forma, a maneira de apresentação da informação fica centrada no modelo cartesiano de descrição da doença e não valoriza as questões sociais e a integralidade do enfermo (Echer, 2005; Starfield, 2002; Vieira; Matias; Queiroz, 2021). Por essa razão ter acesso às informações claras e confiáveis, sobre as enfermidades com as quais convive, é uma ferramenta importante para prevenção e terapêutica das doenças, permitindo uma maior longevidade, com qualidade de vida (Oliveira \& Cota, 2018). Assim objetivou-se pesquisar, através de aplicação de questionário, a temática de eleição, pela clientela, para a ação de EPS, e verificar se o contato com a doença, influencia no interesse de busca por conhecimentos sobre a mesma. Embora os gastos com saúde e assistência social sejam frequentemente vistos como custos para a sociedade, devem ser entendidos como investimentos na concretização de oportunidades e na capacitação individual para que continuem a dar suas muitas contribuições positivas. Falhas no desenvolvimento de políticas sólidas podem prejudicar significativamente a qualidade da saúde e da assistência social que o indivíduo recebe. Dados da Organização Mundial de Saúde revelam que a maioria da população mundial viverá até os 60 anos ou mais. Uma vida mais longa representa a chance de buscar novas atividades, enquanto continua a fazer contribuições valiosas para a família e a comunidade. No entanto, a extensão dessas oportunidades depende muito do fator saúde.

\section{Metodologia}

Tendo em vista os objetivos desta pesquisa, a mesma classifica-se como exploratória, descritiva, com abordagem qualiquantitativa, a qual foi desenvolvida no Ambulatório Sette de Barros (ASB) aberto à participação da comunidade da área de abrangência das Estratégias Saúde da Família (ESF) Sette de Barros I e Sette de Barros II, na cidade de Ponte Nova, Minas Gerais, Brasil, que, de acordo com o DATASUS, o total de pacientes atendidos correspondem a, aproximadamente, 5.562 pessoas (Brasil, 2015).

O estudo foi realizado através da coleta de dados, por meio de um questionário impresso, seguindo um roteiro semiestruturado com seis perguntas objetivas, com abordagem de todas as pessoas que chegavam até a unidade, à procura de um dos serviços prestados pelo serviço de saúde e enquanto aguardavam pelo mesmo. Os usuários do ASB foram convidados à participação na pesquisa, de forma voluntária, após a leitura, compreensão e deferimento do Termo de Consentimento Livre e Esclarecido (TCLE).

Para estabelecer o tamanho da amostra para a aplicação do questionário realizou-se um cálculo de amostra finita, com nível de confiança de 95\% e margem de erro de 5\%, com estimativa de perda de 20\%, por não atendimento aos questionários. Nas tardes de quarta-feira, nos meses de setembro e novembro de 2018 foram aplicados 378 questionários.

O estudo foi realizado mediante a aprovação do Comitê de Ética em Pesquisa (CEP) da Faculdade Dinâmica do Vale do Piranga, com número de parecer 2.925.286, em atendimento à orientação da Resolução 466/12 do Conselho Nacional de Saúde (CNS) uma vez que a pesquisa envolveu seres humanos.

Após coleta e tabulação dos dados, foi realizada a análise descritiva dos mesmos. As variáveis categóricas foram expressas em frequências absolutas e relativas e as variáveis quantitativas foram expressas como média e desvio padrão. O software utilizado foi o Epi Info versão 3.5.1.

\section{Resultados e Discussão}

Os dados obtidos a partir da análise do questionário aplicado aos usuários da área de abrangência do ambulatório Sette de Barros, um serviço de Atenção Primária à Saúde (APS), no município de Ponte Nova, Minas Gerais estão representados 
apresentados no Quadro 1.

Quadro 1 - Análise do questionário aplicado aos usuários da área de abrangência do ambulatório Sette De Barros, no município de Ponte Nova, Minas Gerais.

\begin{tabular}{|c|c|}
\hline Nível de escolaridade & $\begin{array}{l}\text { Curso primário/ Admissão }-111^{\mathrm{x}} \\
\text { Ensino fundamental - } 43^{\mathrm{x}} \\
\text { Ensino médio/ Curso ginasial }-190^{\mathrm{x}} \\
\text { Ensino superior - 22 } \\
\text { Pós-graduação - } 7^{\mathrm{x}} \\
\text { Não informou - } 5^{\mathrm{x}}\end{array}$ \\
\hline $\begin{array}{l}\text { Tempo de espera por consulta, após a chegada ao } \\
\text { ASB }\end{array}$ & $\begin{array}{l}15 \text { minutos }-163^{x} \\
30 \text { minutos }-196^{x} \\
1 \text { hora }-19^{x}\end{array}$ \\
\hline Temas de interesse para discussão ${ }^{\mathrm{bc}}$ & $\begin{array}{l}\text { Câncer de mama }-183^{\mathrm{x}} \\
\text { Depressão - } 292^{\mathrm{x}} \\
\text { Diabetes - } 187^{\mathrm{x}} \\
\text { Dislipidemia - } 256^{\mathrm{x}} \\
\text { Higienização de alimentos }-87^{\mathrm{x}} \\
\text { Hipertensão arterial - } 261^{\mathrm{x}} \\
\text { Insônia - } 247^{\mathrm{x}} \\
\text { Osteoporose - } 149^{\mathrm{x}} \\
\text { Outros - } 63^{\mathrm{x}} \\
\end{array}$ \\
\hline \multirow{7}{*}{$\begin{array}{l}\text { Contato com pessoas que apresentem algum(s) dos } \\
\text { problemas listados }{ }^{c}\end{array}$} & Câncer de mama $-119^{x}$ \\
\hline & Depressão $-291^{x}$ \\
\hline & Diabetes $-287^{x}$ \\
\hline & Dislipidemia $-65^{\mathrm{x}}$ \\
\hline & Hipertensão arterial - $311^{x}$ \\
\hline & Insônia - $289^{x}$ \\
\hline & Osteoporose $-63^{x}$ \\
\hline
\end{tabular}

${ }^{a}$ ASB: ambulatório Sette de Barros. ${ }^{b}$ Indagação realizada apenas aos usuários que consideravam o momento de sala de espera oportuno para participar de roda de conversa. ${ }^{c}$ Os entrevistados poderiam escolher mais de uma opção. ${ }^{x}$ Número de respostas dadas pelas pessoas que responderam aos questionários. Fonte: Autores (2021).

Do universo de entrevistados (378), na primeira coleta de dados, a maioria estudou até o ensino médio (138), o que demonstra uma diferença com o trabalho de Guibuni et al. (2017), o qual realizou uma pesquisa com usuários do serviço de atenção primária, maiores de 17 anos de idade, que frequentam 1541 unidades básicas de saúde (UBS) no Brasil, revelando que mais da metade dos entrevistados estudaram até o ensino fundamental.

Quanto ao tempo em que esperam, em média, para o atendimento, 215 usuários do serviço de saúde aguardam durante 30 minutos ou mais, corroborando com o estudo de Gomide et al. (2018), que demonstrou a longa espera por um atendimento na APS, em um estudo feito em cinco unidades de pronto-atendimento no município de Ribeirão Preto - SP, cuja intenção era avaliar o porquê do grande número de pessoas procurarem por ações de saúde no nível secundário e não no primário e, no qual, foi verificado que a maioria dos clientes aguardam, no mínimo, 1 hora, enquanto esperam por assistência da equipe multidisciplinar na APS.

Com relação ao tema eleito para discussão durante a roda de conversa, 292 usuários escolheram a temática depressão com está representado na Figura 1 - condição clínica que caracteriza-se por um quadro de sintomas intensos, em que, necessariamente, devem estar presentes anedonia e/ou humor polarizado para estado de tristeza e 4 ou mais dos seguintes critérios, 
por no mínimo, dois meses (Fontana, 2005): (i) hiperfagia ou perda de apetite; (ii) insônia ou hipersonia; (iii) agitação ou lentificação psicomotora; (iv) fadiga ou perda de energia - astenia; (v) sentimento de inutilidade ou culpa excessiva ou inadequada e (vi) pensamentos de morte recorrentes e/ou ideação suicida e/ou tentativa de suicídio.

Figura 1 - Gráfico ilustrando a elegibilidade de temas pelos usuários do ambulatório público.

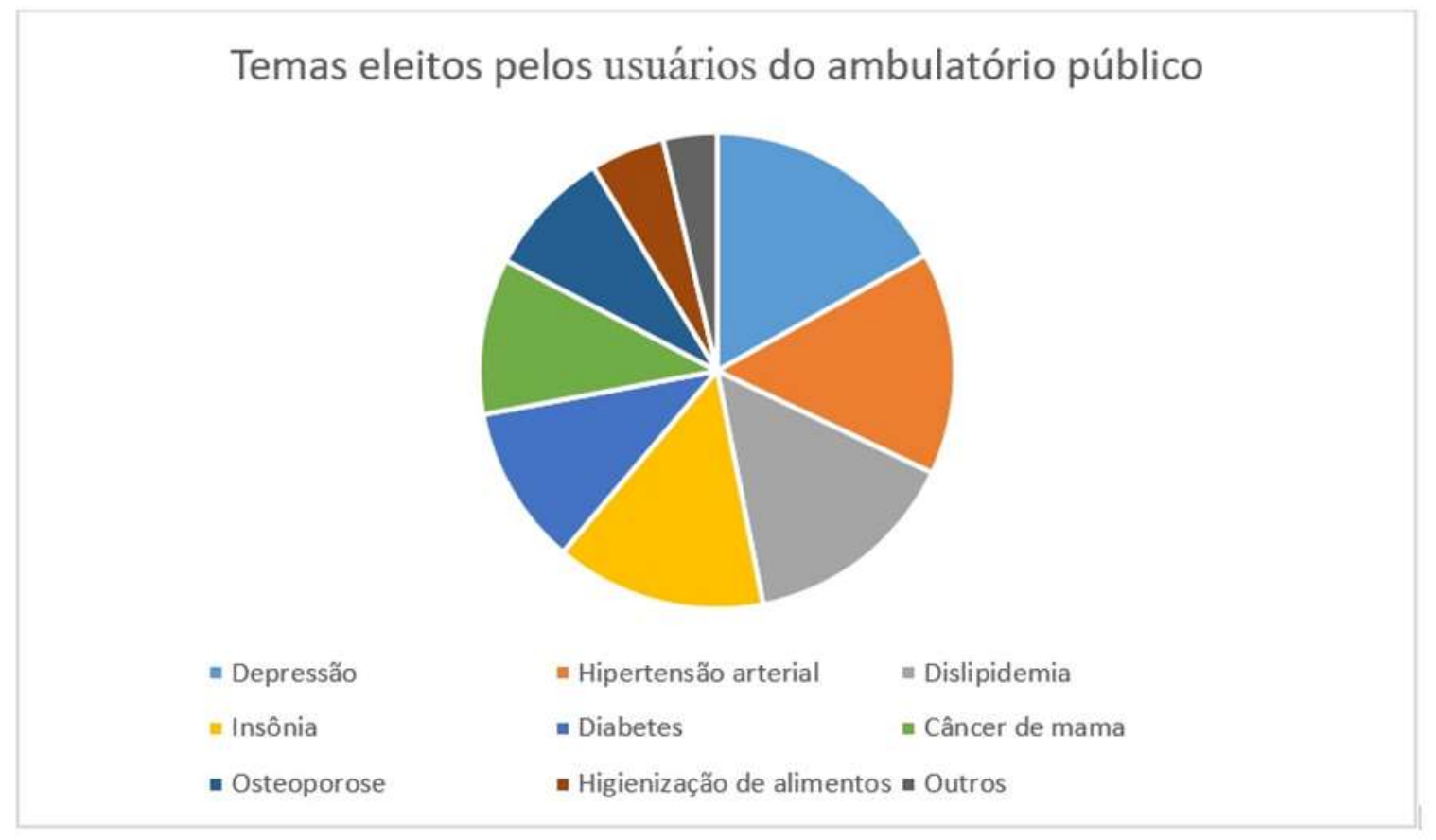

Fonte: Autores (2021).

Quando tratados com antidepressivos e psicoterapia, 80\% dos casos de depressão apresentam remissão dos sinais e sintomas do quadro clínico (Del Porto, 1993).

Pode-se afirmar que a preferência pela temática se justifica pelo fato de que a depressão em estágios mais avançados consiste, atualmente, no fator causal mais importante de inaptidão no mundo, abrangendo o quarto de dez lugares, na avaliação das doenças com maiores agravos de saúde e de acordo com as estimativas, chegará ao segundo lugar nos próximos vinte anos. Na Europa, em um estudo de 2008, foi verificado que o transtorno depressivo é igual a 6,2\% dos problemas de saúde da região (Apóstolo et al., 2008). No Brasil, em ESF, aproximadamente 5\% a 10\% da população adscrita apresenta depressão, o que demanda maior atenção voltada a este grupo de pacientes (Costa, 2015).

$\mathrm{Na}$ presente pesquisa, todos os participantes conhecem pessoas, que apresentam algum dos acometimentos de saúde listados: hipertensão arterial, diabetes, dislipidemia, insônia, colesterol, depressão, câncer de mama e/ou osteoporose. A maioria dos entrevistados demonstrou ter contato com alguém que apresenta hipertensão arterial, seguido de depressão. Esses dados se encontram representados na Figura 2. Além disso, no trabalho, verificou-se que a hipertensão arterial foi a segunda temática escolhida para o debate nas rodas de conversa. Contudo, optou-se por falar em depressão, porque além de ter sido o tema de eleição, a maioria das pessoas tem a necessidade de conhecimento sobre uma doença mental, que ainda traz muito estigma, dúvidas, adquirindo um caráter desprezível e preconceituoso para grande parte dos usuários. Em contrapartida, a hipertensão é um tema mais debatido, por exemplo, nos grupos de hipertensos, nas campanhas, em materiais explicativos e meios midiáticos de divulgação de informação. Entretanto, é válido ressaltar que ambas condições clínicas - depressão e hipertensão - requerem o acompanhamento longitudinal - ou seja, ao longo do tempo, necessitando de acompanhamento por mais de um atendimento em saúde, com abordagem transdisciplinar na ESF (Gusso, 2018; López et al., 2018). 
Figura 2 - Gráfico representando as enfermidades com as quais os entrevistados referiram conviver.

\section{Enfermidades com as quais os entrevistados convivem}

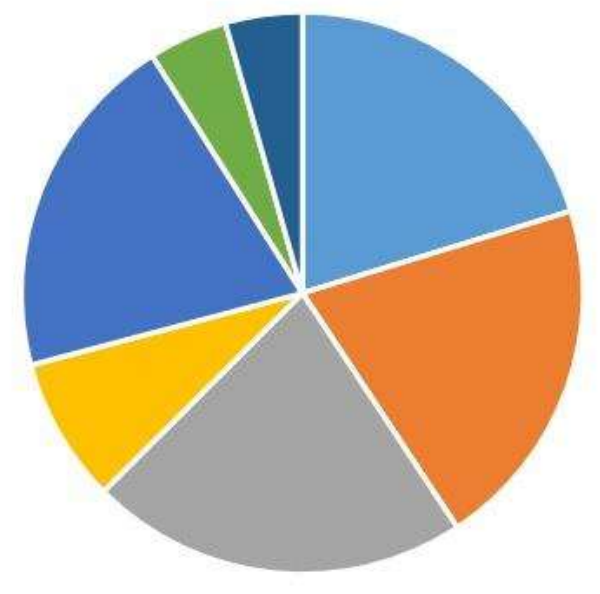

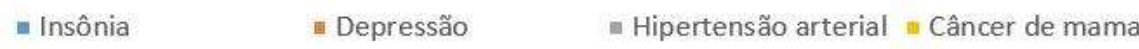

$$
\begin{aligned}
& \text { - Diabetes } \quad \text { = Dislipidemia Osteoporose }
\end{aligned}
$$

Fonte: Autores (2021).

\section{Considerações Finais}

A pesquisa identificou que: (i) com relação ao tema eleito para discussão o mais votado foi depressão com 292 votos; seguido por hipertensão arterial (261); dislipidemia (256) e insônia 247; (ii) a maioria dos entrevistados demonstrou ter contato com alguém que apresenta hipertensão arterial (311 respondentes), seguido de depressão (291 optantes), insônia (289) e diabetes (287).

Pode-se afirmar que há uma relação positiva entre escolha pela temática e a vivência dos pacientes com as doenças. Algumas doenças já possuem tratamento eficaz e informações amplamente divulgadas como a hipertensão arterial e diabetes. Doenças como depressão e insônia têm apresentado crescimento nas últimas décadas (Costa, 2015), justificando o interesse da população por informações sobre esses temas.

Ao conviver com determinadas patologias os indivíduos apresentam dúvidas sobre o tema e a Atenção Primária mostrase como um dos locais de busca por informações seguras e acessíveis sobre temáticas relacionadas a saúde.

\section{Referências}

Apóstolo, J., Ventura, A., Caetano, C. \& Costa, S. (2008) Depressão, ansiedade e stresse em utentes de cuidados de saúde primários. Coimbra: Escola Superior de Enfermagem de Coimbra. 46p. https://www.scielo.br/pdf/rlae/v19n2/pt_17.pdf

Brasil. DATASUS CNES. (2015). http://datasus.saude.gov.br/cadastro-nacional-de-estabelecimentos-de-saude.

Costa, T. S. (2015). Rastreamento de sintomas depressivos em usuários assistidos pela Estratégia de Saúde da Família em um município de pequeno porte no nordeste brasileiro [tese]. São Paulo: Faculdade de Ciências Médicas da Santa Casa de São Paulo. 98f. http://ojs.unesp.br/index.php/ revista_proex/oai?verb=ListRecords\&set=revista_proex\&metadataPrefix=oai_dc

Del Porto, J. A. (1993). Depressões. In: Ramos O. L, Rot-Schild H. A. Atualização terapêutica. (16a ed.), Artes Médicas. 949-951 e 1171-1172.

Echer, I. C. (2005) Elaboração de manuais de orientação para o cuidado em saúde. Rev Lat Am Enferm. 13(5), 754-757.

Ferreira, L., Barbosa, J. S. A., Esposti, C. D. D. \& Cruz, M. M. (2019A). Educação Permanente em Saúde na atenção primária: uma revisão integrativa da literatura. Saúde debate. 43:120, 223-239. 
Ferreira, M. J. M., Ribeiro, K. G., Almeida, M. M., Sousa, M. S., Ribeiro, M. T. A. M., Machado, M. M. T. \& Kerr, L. R. F. S. (2019B). Novas Diretrizes Curriculares Nacionais para os cursos de Medicina: oportunidades para ressignificar a formação. Interface, Botucatu. 23(1).

Fontana, A. M. Manual de Clínica em Psiquiatria. Editora Atheneu, 2005.

Gomide, M. F. S., Pinto, I. C., Bulgarelli, A. F., Santos, A. L. P. \& Gallardo, M. P. S. (2018). A satisfação do usuário com a atenção primária à saúde: uma análise do acesso e acolhimento. Interface. Botucatu, 22(65), 387-398.

Guibuni, I. A., Moraes, J. C., Guerra Junior, A. A., Costa, E. A., Acurcio, F. A., Costa, K. S., Karnikowski, M. G. O., Soeiro, O. M., Leite, S. N. \& Álvares, J. (2017). Características principais dos usuários dos serviços de atenção primária à saúde no Brasil. Revista de saúde Pública. 17(2).

Gusso, G. \& Lopes, J. M. C. (2018). Tratado de Medicina de Família e Comunidade. Artmed, 2

Kugbey,N., Meyer-Weitz, A. \& Asante, K. O. (2019) Acesso a informações sobre saúde, alfabetização em saúde e qualidade de vida relacionada à saúde entre mulheres que vivem com câncer de mama: depressão e ansiedade como mediadores. National Library of Medicine. 102 (7), $1357-1363$.

López, M. M., Mohrbach, D. Y., Puyana, C. R., Anderson, M. I. P., Mejía, P. A. C., Fortes, S., Quiroz, J. R. \& González, M. L. V. (2018) La Medicina Familiar y Comunitaria como fuente de cuidados de Salud Mental. Revista Brasileira de Medicina de Família e Comunidade. 13(10), 54-68.

Liu, P. L., \& Jiang, S. A. (2019). Comunicação centrada no paciente medeia a relação entre a aquisição de informações de saúde e a confiança do paciente nos médicos: uma comparação de cinco anos na China. Comunicação em saúde. 36(2).

Moretti-Pires, R. O., \& Bueno, S. M. V. (2009). Freire e formação para o Sistema Único de Saúde: o enfermeiro, o médico e o odontólogo. Acta Paul Enferm. 22(44), 39-44.

Oliveira, M. F., \& Cota, L. G. S. (2018). A pedagogia freiriana nas práticas de educação em saúde. Diversitates International Journal, 10(1), 46-58.

Oliveira, S. F., \& Machado, F. C. A. (2020). Percepção dos profissionais da Estratégia Saúde da Família sobre processos educativos em saúde. Revista Ciência Plural. 6(1), 56-70.

Pereira, A. S. et al. (2018). Metodologia da pesquisa científica. Biblioteca Central da UFSM. https://repositorio.ufsm.br/bitstream/handle/1/15824/Li c_Computacao_Metodologia-Pesquisa-Cientifica.pdf?sequence=1

Organização Mundial da Saúde (OMS) (2017). 10 fatos sobre envelhecimento e saúde. https://www.who.int/news-room/fact-sheets/detail/10-facts-on-ageingand-health

Starfield, B. (2002) Atenção primária: equilíbrio entre necessidades de saúde, serviços e tecnologia. Brasília: UNESCO, Ministério da Saúde, 2002. http://www.dominiopublico.gov.br/download/textoue000039.pdf

Vieira, M. S. N., Matias, K. K. \& Queiroz, M. G. (2021). Educação em saúde na rede municipal de saúde: práticas de nutricionistas. Ciênc. Saúde Colet. 26(02). 